\title{
Prävention und Früherfassung von Kindsmisshandlung - ein Gebot der Stunde
}

Ulrich Lips

Dr. med., Facharzt FMH Kinder- und Jugendmedizin, Leiter Kinderschutzgruppe und Opferberatungsstelle,

Universitäts-Kinderkliniken Zürich
* Die Literaturangaben finden sich im Internet unter www.saez.ch $\rightarrow$ Aktuelle Nummer oder $\rightarrow$ Archiv $\rightarrow 2011$ $\rightarrow 11$.

Korrespondenz:

Dr. med. Ulrich Lips

Kinderschutzgruppe und Opferberatungsstelle

Universitäts-Kinderkliniken Steinwiesstrasse 75

CH-8032 Zürich

ulrich.lips@kispi.uzh.ch

www.kinderschutzgruppe.ch
In Kürze werden in der Schweiz 20000 Ärztinnen und Ärzte verschiedener Fachgruppen kostenlos eine neue Broschüre mit dem Titel «Kindsmisshandlung - Kindesschutz» erhalten. Hinter der Präventionsoffensive stehen die Stiftung Kinderschutz Schweiz als Herausgeberin und der Autor Ulrich Lips, Leiter der Kinderschutzgruppe und Opferberatungsstelle des Kinderspitals Zürich. Der nеuе Leitfaden für Ärztinnen und Ärzte sowie deren Praxisassistentinnen dient der Früherkennung von Kindsmisshandlung und dem Vorgehen in der ärztlichen Praxis. Er wird von der FMH sowie zahlreichen Fachgesellschaften und -verbänden unterstützt.

\section{Ansteigende Fallzahlen und ihre Bedeutung} Die Kinderschutzgruppe und Opferberatungsstelle des Kinderspitals Zürich verzeichnete im Jahr 2010 einen Anstieg der gemeldeten Fälle von Kindsmisshandlung um 16\%. Die dokumentierten 487 Fälle stellen die bedenkliche Höchstmarke seit den Erhebungen im Jahre 1969 dar.

Am häufigsten gemeldet werden Fälle von vermuteter oder sicherer sexueller Ausbeutung, gefolgt von körperlichen Misshandlungen, psychischer Misshandlung und Vernachlässigung. Die Verteilung der Misshandlungsformen ist in Abbildung 1 dargestellt.
Die Zunahme der Meldungen von Kindsmisshandlung hat verschiedene Facetten: Zum einen wird klar, dass Kindsmisshandlung ein häufiges Phänomen ist. Zusätzlich zum Kinderspital gibt es im Kanton Zürich noch mehr als ein Dutzend weiterer Anlaufstellen, bei denen die Misshandlung eines Kindes gemeldet werden kann, und überall steigen die Zahlen an. Eher vorsichtige internationale Schätzungen gehen denn auch davon aus, dass 10 bis $20 \%$ der Kinder bis zu ihrem 18. Geburtstag Opfer von Misshandlungen werden [1] ${ }^{*}$. Zahlen aus sämtlichen Schweizer Kinderkliniken werden im Frühsommer 2011 publiziert werden.

Dabei ist man sich einig, dass das Phänomen Kindsmisshandlung nicht zunimmt, vielmehr entspricht die steigende Zahl der Meldungen einer höheren Aufdeckungsrate: Fach- und Bezugspersonen von Kindern achten offenbar in vermehrtem Masse auf die vielfältigen Symptome von Kindsmisshandlung und machen den wichtigen ersten Schritt, mit ihren Beobachtungen an eine Fachstelle zu gelangen. Höhere Fallzahlen bedeuten also auch, dass die Dunkelziffer von Kindsmisshandlung abnimmt. Das ist an sich erfreulich, denn nur erkannten Opfern kann geholfen werden.

\section{Abbildung 1}

Misshandlungsformen der gemeldeten Fälle 2010, Kinderspital Zürich.

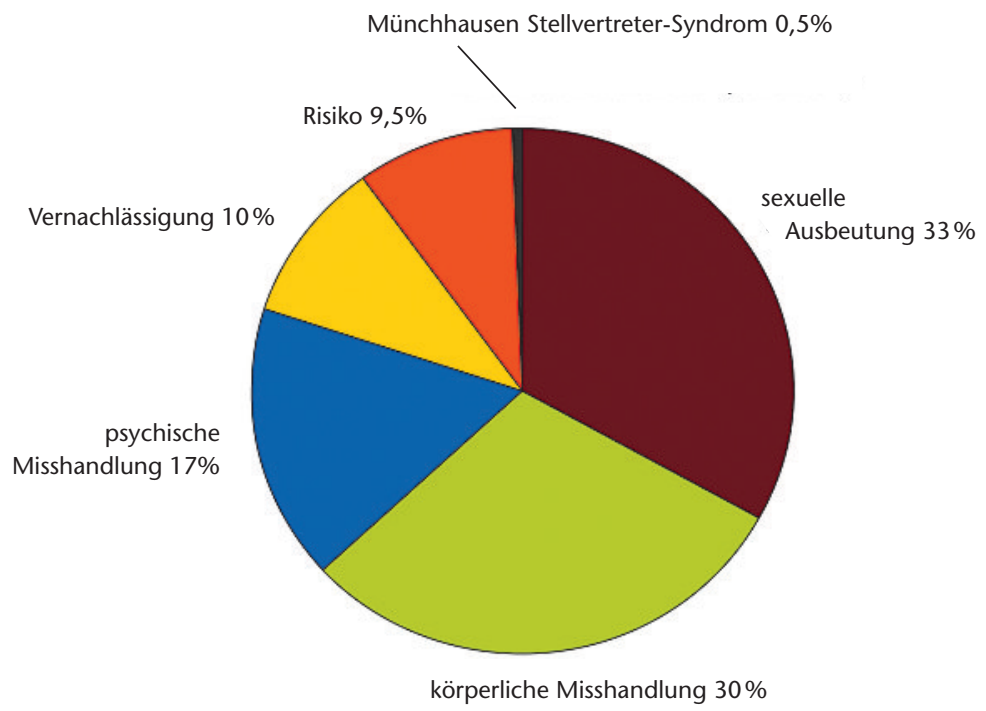

$n=487$ 
Tabelle 1

Folgen von Adverse childhood experiences (ACE) im Erwachsenenalter. (Modifiziert nach [7, 8]).

Problem

Zusammenhang mit ACE

++ starke Korrelation + mässige Korrelation

Keine Berufsausbildung

Alkoholabusus

Depression

Drogenabusus

Posttraumatische Belastungsstörung

Selbstverletzendes Verhalten

Suizidversuche

Kriminelles Verhalten

Prostitution

Anzahl Hospitalisationen allgemein

Hohe Gesundheitskosten

Adipositas

Asthma

Autoimmunerkrankungen

Diabetes

Herzinfarkt

Hirnschlag

Migräne

Spastische Colitis

$+$

$++$

$++$

$++$

$++$

$++$

$++$

$++$

$+$

$+$

$++$

$++$

$+$

$++$

$++$

\section{Childhood adverse experiences und ihre Folgen}

Grosse Studien zeigen, dass traumatische Ereignisse im Kindesalter (childhood adverse experiences) weitreichende Auswirkungen auf die spätere psychische und physische Gesundheit, das Sozialverhalten und die Berufskarriere des betroffenen Individuums haben [2-10]. Die häufigste Gruppe von childhood adverse experiences ist die Kindsmisshandlung, ebenfalls dazu gehören Tod von nahen Bezugspersonen, Trennung der Eltern, Kriegserlebnisse und Naturkatastrophen. Tabelle 1 gibt einen Überblick über die in der Literatur berichteten Folgezustände. Daraus wird klar, dass Kindsmisshandlung zusätzlich zum individuellen Leid unermessliche gesellschaftliche Auswirkungen hat und hohe Aufwendungen des Gesundheitswesens verursacht.

\section{Ärztinnen und Ärzte können etwas bewirken}

Trotz verbesserter Aufdeckungsrate ist die Dunkelziffer von Kindsmisshandlung mit Sicherheit noch sehr gross, insbesondere bei sexueller Ausbeutung, psychischer Misshandlung und Vernachlässigung. Diese misshandelten Kinder sehen wir Ärztinnen und Ärzte täglich in der Praxis, in der Sprechstunde und im Spital: wegen Beschwerden, Krankheiten und Unfällen, zur Vorsorgeuntersuchung und zum Impfen. Und wir sehen auch ihre Eltern: mit psychischen Erkrankungen, chronischen Krankheiten, mit Paarkonflikten und nach häuslicher Gewalt, d. h. mit Risikofaktoren für die Misshandlung ihrer Kinder.

Es muss das erklärte Ziel unserer Gesellschaft sein, die Dunkelziffer von Kindsmisshandlung zu senken.
Wir Ärztinnen und Ärzte haben die einmalige Chance dazu: $\mathrm{Zu}$ uns kommen Patientinnen und Patienten mit ihren Sorgen und Nöten, mit intimsten Details aus ihrem Leben, mit Beschwerden und Verletzungen, deren Ursachen sie nur im Rahmen höchster Vertraulichkeit aufdecken. Und sie bringen uns ihre Kinder, deren Entwicklung wir begleiten. In diesem Rahmen können wir viele Signale empfangen und Symptome bemerken, die eine Gefährdung des Kindswohls bedeuten. Dabei müssen wir unsere privilegierte Stellung in aller Regel nicht gefährden - das Gesetz verbietet es uns ja auch, das Patientengeheimnis $\mathrm{zu}$ verletzen. Eltern möchten für ihre Kinder das Beste, mit ganz wenigen Ausnahmen. Gelegentlich sind sie aber nicht in der Lage, einschätzen oder geben zu können, was das Beste für ihr Kind ist. Deshalb sind sie uns Ärzten meistens dankbar, wenn wir ihre Signale aufnehmen, nachfragen, Interesse am Wohl ihrer Kinder zeigen. Wenn das Kindswohl allerdings klar gefährdet oder gar bereits beeinträchtigt ist, erlaubt es uns die rechtliche Situation auch, ohne Einverständnis der Eltern eine Gefährdungsmeldung bei der Kinder- und Erwachsenenschutzbehörde zu deponieren; in einigen Kantonen besteht sogar eine Meldepflicht.

Ein Instrument gegen die Hilflosigkeit...

Grosse Umfragen ergeben, dass sich die meisten Ärztinnen und Ärzte aber in der Diagnostik von Kinds-

\section{Kurzinformationen zur Broschüre «Kinds- misshandlung - Kindesschutz - Ein Leitfaden zu Früherfassung und Vorgehen in der ärztli- chen Praxis"}

Herausgeberin: Stiftung Kinderschutz Schweiz. Autor: Dr. med. Ulrich Lips, Klinischer Dozent, Leiter der Kinderschutzgruppe und Opferberatungsstelle Universitäts-Kinderkliniken Zürich.

Zielgruppe: Ärztinnen und Ärzte der folgenden Fachrichtungen und deren Praxisassistentinnen: Kinderärzte, Grundversorger, die Kinder betreuen, Kinder- und Jugendpsychiater, Kinderchirurgen, Chirurgen, Dermatologen, HNO-Ärzte, Geburtshelfer, Gynäkologen, Neonatologen, Zahnärzte, andere Ärztinnen und Ärzte, die keine Kinder betreuen.

Sprachen: gebunden in Deutsch und Französisch, als pdf-Download in Deutsch, Französisch, Italienisch.

Erhalt: Der Leitfaden wird in der letzten Märzwoche 2011 an 20000 Ärzte und Ärztinnen der oben genannten Fachrichtungen geschickt. Darüber hinaus kann die Broschüre in Deutsch, Französisch und Italienisch auf www.kinderschutz.ch heruntergeladen werden. Für Fragen steht die Stiftung Kinderschutz Schweiz gerne zur Verfügung: Telefon: 031/ 3981010 / E-Mail: info@ kinderschutz.ch 
misshandlung unsicher und im Umgang damit hilflos fühlen $[11,12]$. Da erstaunt es nicht, dass man gerne einmal die Schultern zuckt oder gar die Augen verschliesst. Um dieser Unsicherheit und Hilflosigkeit entgegenzuwirken, gibt die Stiftung Kinderschutz Schweiz einen Leitfaden heraus, der einen kurzen Überblick über das Gebiet gibt und aufzeigt, wie in einem Verdachtsfall vorgegangen werden kann. Ziel und Grundprinzip des Kinderschutzes («Nie allein») werden ebenso erläutert wie der konkrete Umgang mit dem Verdacht in der Praxis (Dokumentation, Kontaktaufnahme mit einer Fachgruppe, weiteres Vorgehen). Zudem wird die rechtliche Situation in der Schweiz (von Kanton zu Kanton verschieden) dargelegt und die Stellung der Ärztinnen und Ärzte ausgeführt. In spezifischen Kapiteln werden für Grundversorger mit und ohne direkten Kontakt zu Kindern sowie für Fachärzte verschiedener Fachrichtungen diagnostische sowie differentialdiagnostische Hinweise gegeben.

\section{...nutzen Sie es!}

Die Broschüre «Kindsmisshandlung - Kindesschutz. Ein Leitfaden zu Früherfassung und Vorgehen in der ärztlichen Praxis» der Stiftung Kinderschutz Schweiz wird Ihnen demnächst über die FMH zugestellt. Sie wird von der FMH und vielen Fachgesellschaften und -verbänden unterstützt. Werfen Sie einen Blick hinein: Für die Lektüre des allgemeinen Überblicks und des Sie betreffenden Kapitels brauchen Sie maximal zehn Minuten. Und wenn bei Ihnen das nächste Mal die Glocke «Kindsmisshandlung?» klingelt, ziehen Sie den Leitfaden wieder hervor - und tun Sie etwas zum Wohl des Kindes und seiner Eltern.

\section{Interview mit Jacqueline Fehr, Präsidentin der Stiftung Kinderschutz Schweiz «Ärztinnen und Ärzte spielen eine entscheidende Rolle»}

Interview: Cordula Sanwald
Ende März wird die Stiftung Kinderschutz Schweiz ihren neuen Leitfaden «Kindsmisshandlung - Kindesschutz» in deutscher und französischer Sprache an 20000 Ärztinnen und Ärzte unterschiedlicher Fachrichtungen in der Schweiz verschicken (siehe dazu den vorangehenden Artikel). Nationalrätin Jacqueline Fehr, Präsidentin der Stiftung Kinderschutz Schweiz, erläutert die Motivation von Kinderschutz Schweiz, einen solchen Ratgeber für Ärztinnen und Ärzte sowie Medizinische Praxisassistentinnen herauszugeben:

Frau Fehr, das Ausmass von Gewalt an Säuglingen und Kleinkindern ist gross. Wovon sprechen wir, wenn wir von Kindsmisshandlung reden?

Jacqueline Fehr: Kindsmisshandlung hat verschiedene Gesichter. Dazu gehört die sexuelle Ausbeutung und körperliche Misshandlung ebenso wie die Vernachlässigung oder psychische Gewalt. Meist liegen die Gründe in der mannigfachen Überforderung von Eltern. Heute weiss man, dass der Ort, an dem Kinder am meisten Gewalt erleben, die Familie ist. Ihre psychische und körperliche Gesundheit ebenso wie ihr Sozialverhalten werden dadurch massiv beeinträchtigt. Das in einem Land, welches sich mit der Unterzeichnung der Kinderrechtskonvention dazu verpflichtet hat, Kinder vor jeder Form körperlicher oder geistiger Gewaltanwendung oder Misshandlung, vor Verwahrlosung oder Vernachlässigung, vor schlechter Behandlung oder Ausbeutung zu schützen.

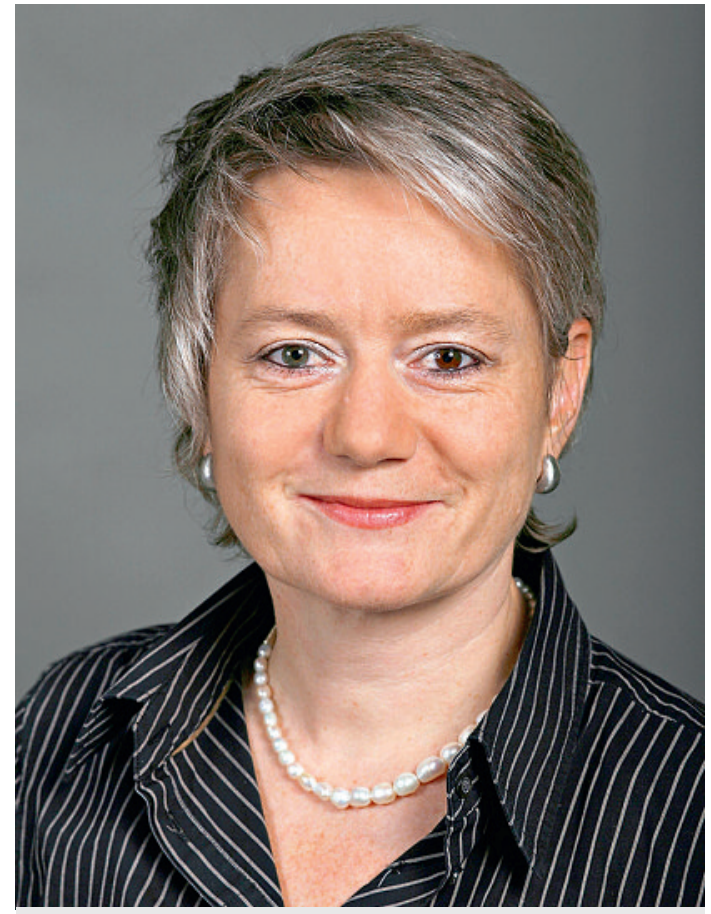

«Einen aktiven Schritt zu tun in einer komplexen Situation wie vermuteter Kindsmisshandlung, braucht viel Mut»: Jacqueline Fehr, Präsidentin der Stiftung Kinderschutz.

Nun will die Stiftung Kinderschutz Schweiz mit einem neuen Leitfaden für Ärzte und Ärztinnen dazu beitragen, die Dunkelziffer zu erhellen. 


\section{Stiftung Kinderschutz Schweiz}

Als nationale Stiftung macht sich Kinderschutz Schweiz dafür stark, dass alle Kinder in unserer Gesellschaft in Würde und ohne die Verletzung ihrer Integrität aufwachsen. Die Stiftung Kinderschutz Schweiz setzt sich deshalb seit Jahren erfolgreich für die Rechte von Kindern und gegen jede Form von Gewalt an Kindern ein. Nicht nur mittels verschiedener Präventionsprojekte wie beispielsweise dem Kinderparcours für Primarschulen «Mein Körper gehört mir!» gegen sexuelle Gewalt, sondern auch durch gezielte politische Lobbyarbeit, Beratung, Netzwerk- und Öffentlichkeitsarbeit. Mit ihrer Fachstelle ECPAT Switzerland (End Child Prostitution, Child Pornography and Trafficking of Children for Sexual Purposes) engagiert sich die Stiftung darüber hinaus in der internationalen Präventionsarbeit gegen Kinderprostitution, Kinderpornografie und Kinderhandel. ECPAT Switzerland arbeitet dabei eng mit Behörden sowie staatlichen und nicht-staatlichen Institutionen zusammen. Weitere Informationen: www.kinderschutz.ch

Wir sind eine nationale Stiftung, die sich dafür einsetzt, dass Kinder mit Würde und unter Wahrung ihrer Rechte und Integrität aufwachsen. Vor dem Hintergrund der schwerwiegenden, negativen Auswirkungen von Kindsmisshandlung auf das spätere Erwachsenenleben gewinnt zunehmend die Prävention und Früherfassung an Bedeutung. Ärztinnen und Ärzte spielen dabei eine entscheidende Rolle. Der Leitfaden soll den Fachleuten Sicherheit in ihrem Handeln geben. Einen aktiven Schritt zu tun in einer komplexen Situation wie vermuteter Kindsmisshandlung, braucht viel Mut. Je grösser die Sicherheit, dass man das Richtige richtig tut, umso grösser ist die Bereitschaft, dieses Wagnis einzugehen. Dabei gilt das Augenmerk der Qualität der Meldungen. Sie ist ausschlaggebend, um Kindsmisshandlungen früh festzustellen. Um diese zu gewährleisten, ist es essentiell, dass für Fachpersonen, die mit Kindern und Eltern zu tun haben, gezielte Informationen bereitgestellt werden.

Informationen, wie beispielsweise?

Zum Beispiel, wann ein begründeter Verdachtsfall besteht oder eine Abklärung bei einer Kinderschutzgruppe Sinn macht. Aber auch, welche Rollen die involvierten Fachpersonen haben und wo die Abgrenzung zur Arbeit von Kindesschutzgruppen, Opferberatungsstellen und Kindesschutzbehörden liegt. Auf diese Fragen gibt der vorliegende Leitfaden präzise Antwort.

Weshalb ist der Leitfaden als Ratgeber für Ärztinnen und Ärzte verschiedenster Fachrichtungen angelegt und nicht nur für Kinderärzte?

Wir sehen an den ansteigenden Meldungszahlen, dass Fachpersonen bereits zunehmend sensibilisiert sind. Diesen Weg möchten wir weitergehen. Unser Leitfaden richtet sich an Ärztegruppen, die in irgendeiner Form mit Kindern und deren Eltern in Berührung kommen. Sie nehmen eine Schlüsselfunktion für die Entwicklung gefährdeter Kinder ein. Wenn sie früh und kompetent reagieren können, ist die Chance gross, dass das Kind im Fall einer vermuteten oder faktischen Kindswohlgefährdung den notwendigen Schutz erfährt. Und - nicht zu vergessen - auch die Eltern Hilfestellung bekommen, um Risikosituationen und Gefährdungen abzuwenden.

Die FMH und andere Fachgesellschaften und -verbände unterstützen den Leitfaden.

Wir sind sehr glücklich über diese Unterstützung. Mit Dr. Lips haben wir einen unermüdlichen Fachmann auf dem Gebiet des Kindesschutzes an unserer Seite. Seinem Erfahrungsschatz und Fachwissen ist es zu verdanken, dass dieser Leitfaden zustande kommen konnte. Nun hoffen wir, dass sich viele Ärztinnen und Ärzte und ihre Praxisassistentinnen dem Engagement anschliessen und den Leitfaden in ihrer Praxis nutzen. Dafür möchte ich ihnen im Namen der Stiftung Kinderschutz Schweiz schon heute danken. 


\section{Literatur}

1 Trocmé N. Epidemiology of child maltreatment. Child welfare research: Advances for practice and policy. D. Lindsey and A. Shlonsky. Oxford, University Press: 2008;15-24.

2 Brown DW, Anda RF, Tiermeier H, Felitti VJ, Edwards VJ, Croft JB, Giles WH. Adverse Childhood Experiences and the Risk of Premature Mortality. Am J Prev Med. 2009;37(5):389-96.

3 Corso PS, Edwards VJ, Fang X, Mercy JA. Healthrelated quality of life among adults who experienced maltreatment during childhood. Am J Public Health. 2008;98(6):1094-100.

4 Danese A, Moffitt TE, Harrington H, Milne BJ, Polanczyk G, Pariante CM, Poulton R, Caspi A. Adverse childhood experiences and adult risk factors for age-related disease: depression, inflammation, and clustering of metabolic risk markers. Arch Pediatr Adolesc Med. 2009;163(12):1135-43.

5 Douglas KR, Chan G, Gelernter J, Arias AJ, Anton RF, Weiss RD, Brady K, Poling J, Farrer L, Kranzler HR. Adverse childhood events as risk factors for substance dependence: partial mediation by mood and anxiety disorders. Addict Behav. 2010;35(1):7-13.

6 Fergusson DM, Boden JM, Horwood LJ. Exposure to childhood sexual and physical abuse and adjustment in early adulthood. Child Abuse Negl. 2008;32(6):607-19.
7 Gilbert R, Spatz Widom C, Browne K, Fergusson D, Webb E, Janson S. Burden and consequences of child maltreatment in high-income countries. Lancet. 2009;373(9657):68-81.

8 Holly L, Wegman HL, Stetler C. A Meta-Analytic Review of the Effects of Childhood Abuse on Medical Outcomes in Adulthood. Psychosomatic Medicine. 2009;71(8):805-12.

9 Slade EP, Wissow LS. Spanking in Early Childhood and Later Behavior Problems: A prospective Study of Infants and Young Toddlers. Pediatrics. 2004;113(5):1321-30.

10 Zielinski DS. Child maltreatment and adult socioeconomic well-being. Child Abuse Negl. 2009;33(10):661-5

11 Lane WG. Primary care pediatricians' experience, comfort and competence in the evaluation and management of child maltreatment: Do we need child abuse experts? Child Abuse Negl. 2009;33(2):76-83.

12 Starling SP, Heisler KW, Paulson JF, Youmans E. Child Abuse Training and Knowledge: A National Survey of Emergency Medicine, Familiy Medicine, and Pediatric Residents and Program Directors. Pediatrics. 2009;123(4): 595-602 